\title{
Concept for an Integrated PNT-Unit for Maritime Applications
}

\author{
Ralf Ziebold, Zhen Dai, Thoralf Noack, Evelin Engler \\ Institute of Communications and Navigation \\ German Aerospace Centre (DLR) \\ Neustrelitz; Germany \\ Ralf.Ziebold@DLR.de
}

\begin{abstract}
The provision of robust position, navigation and timing (PNT) information is a fundamental element of the e-Navigation initiative proposed by the International Maritime Organization (IMO). In this context the single Electronic Position Fixing System (EPFS), which currently conforms to the minimum carriage requirement, should be replaced by an integrated PNT unit as future onboard integrated system. This unit has the task to collect and integrate data from individual PNT sensors in order to deliver robust PNT information with a specified performance. In this paper the current status of PNT sensors for maritime application is reviewed and first ideas towards an integrity concept for a maritime PNT unit are presented.
\end{abstract}

Keywords: GNSS, PNT, maritime navigation, sensor integration, integrity monitoring

\section{MOTIVATION}

As a consequence of increased maritime traffic and caused incidents, the International Maritime Organization (IMO) has issued the "e-Navigation Strategy" in order to support its vision of "Safe, secure, and efficient shipping on clean oceans" [1].

E-Navigation ( $\mathrm{e}=$ electronic/enhanced) is considered as a strategic framework for developing existing and future technological infrastructure onboard and ashore. It is defined as [1]: the harmonised creation, collection, integration, exchange and presentation of maritime information onboard and ashore by electronic means to enhance berth to berth navigation and related services, for safety and security at sea and protection of the marine environment.

IMO defined the overarching framework for developing the e-Navigation architecture by introducing a strategy for the development and implementation of e-Navigation [2]. It can be divided into three parts: an onboard part, an ashore part and the communication link between both parts. The vision for the onboard part herein is a navigation system that benefits from the integration of own ship sensors, supporting information, a standard user interface, and a comprehensive system for managing guard zones and alerts.
Position fixing has been identified as one of the key strategic elements of e-Navigation and hence should meet user needs in terms of accuracy, integrity, reliability and system redundancy in accordance with the level of risk and volume of traffic.

Following the vision of e-Navigation the International Association of Marine Aids to Navigation and Lighthouse Authorities (IALA) has proposed an integrated e-Navigation PNT device as part of an Integrated Navigational System (INS) [3].

The basic idea is to replace the currently required Electronic Position Fixing System (EPFS) on board of a vessel, which is only based on a single PNT source. We interpret a PNT unit as a system function, which collects and integrates data from individual PNT sensors in order to deliver robust PNT information with a specified performance (e.g. accuracy, integrity). The PNT unit should internally identify and automatically switch to the sensors or sensor combinations delivering the best PNT solution.

The PNT unit should be based on a GNSS core element and aided by appropriate augmentation systems. It should also include interfaces for the utilization of adequate sensors to maintain redundancy, backup or contingency functionality of the system in case of a failure of the primary GNSS sensor.

In a modular concept a PNT unit can be seen as one part of an Integrated Navigational System (INS) which is responsible for delivering the position, navigation and timing information. The INS should use this information in order to perform its system tasks like collision avoidance, route planning and route monitoring.

Following the ideas of the e-navigation strategy this paper will discuss a concept of an integrated PNT unit for maritime applications. In section II, the requirements of a PNT unit will be derived from the relevant user needs. In section III, the current status of standard sensors providing PNT information onboard a vessel will be described. From the comparison between the actual status and the defined requirements, a gap analysis will be performed. Subsequently possible nonstandard sensors providing additional PNT information will discussed in section IV. Finally in section V, we will present 
first ideas towards an integrity concept for a maritime PNT unit.

\section{REQUIREMENTS FOR A MARITIME PNT UNIT}

\section{A. User needs}

The implementation of the e-Navigation strategy starts with the identification of user needs. Therefore different user surveys were conducted and summarized in [4]. Referring to the tasks of a PNT unit, the following three user needs can be identified:

i. Improved reliability: Before mariners can feel confident about relying on systems under the enavigation concept, they must prove far more reliable than many of the present systems.

ii. Indication of reliability

iii. Alert management: Bridge alerts (emergency alarms, alarms, warnings and cautions) must be coordinated, weighted, and support decision making without undue distraction.

Concerning user need (i) the main question is how to make the user feel more comfortable when relying on a PNT system. Therefore the term "reliability" needs to be detailed in measurable parameters, including: a) accuracy, b) integrity, c) continuity d) availability. IMO has given a definition of these parameters for maritime applications. For instance, integrity is defined as the ability to provide users with warnings within a specified time when the system should not be used for navigation. Additionally, an alert limit needs to be defined during integrity monitoring in order to identify the maximum allowable error in position domain before an alarm is triggered. Following this, an integrity monitoring needs to be performed for each output parameter, where the measurement error is estimated and checked against a certain threshold (alert limit). Here it needs to be taken into account, that the accuracy requirements and the alert limit may vary temporally and / or spatially.

User need (ii) is a task of the Human Machine Interface (HMI) rather than a task of the PNT unit. In this case, the PNT unit only provides reliability indicators to the HMI.

With respect to user need (iii), a PNT unit needs a defined interface to the overall alert management system of the vessel and needs to keep tracking the entire sensor systems used within the unit itself. Since a PNT unit comes with functionalities to detect failure of sensors, the unit will include its own PNT alert management.

\section{B. Requirements for a PNT unit}

Based on the identified user needs, the requirements for a maritime PNT unit would need to be drawn. This is actually a task of the maritime organizations. Within this paper we will review existing standards with respect to those requirements and highlight open points.

In [5] IALA has evaluated the GNSS vulnerability and recommends possible mitigation measures. Especially the need of redundant, backup or contingency sources of PNT information is highlighted. The definitions of these terms are given below:

A redundant system provides the same functionality as the primary system, allowing a seamless transition with no change in procedures.

A backup system ensures continuation of the navigation application, but not necessarily with the full functionality of the primary system and may necessitate some change in procedures by the user.

A contingency system allows safe completion of a manoeuvre, but may not be adequate for long-term use.

For the selection of sensors for a PNT unit it is necessary to check which of these system functionalities can be provided.

In the next step the basic output parameters of a maritime PNT unit need to be defined. As a preliminary design, the following parameters should be offered by a PNT unit:

(i) Position: It mainly contains the longitude and latitude for maritime navigations. Because vessels can usually be found close to the sea level, the height information is usually not provided as standard output parameter.

(ii) Under keel clearance (UKC): Instead of the height information, the UKC is the relevant maritime output parameter. It is defined as the distance between the lowest point of the ship (e.g. the keel) and the ground of the sea.

(iii) Velocity: The magnitude and direction of a velocity vector can be described by Speed over Ground (SOG) and Course over Ground (COG). Because of their physical principles, speed sensors like e.g. electro-magnetic logs can only measure the speed through water (STW), and therefore STW is also a parameter which a PNT unit could deliver.

(iv) Attitude: Generally the orientation of the ship in the horizontal plane is reported. Here one needs to distinguish between the orientation with respect to the true north (true heading) and with respect to the magnetic north (heading). For future applications, the other attitude angles, namely roll and pitch, could also be required.

\section{(v) Timing: UTC time needs to be delivered.}

After clarification of the basic output parameters, user requirements concerning accuracy and integrity with respect to the different areas of maritime traffic needs to be defined. Looking into existing standards, the IMO document regarding the requirements for a future GNSS could be interpreted as user requirements for the positioning part of the PNT unit, as shown in Table I.

TABLE I: IMO REQUIREMENTS FOR A FUTURE GNSS [6]

\begin{tabular}{|c|c|c|c|}
\hline & $\begin{array}{c}\text { Ocean/ } \\
\text { Coastal }\end{array}$ & Port & $\begin{array}{c}\text { Automatic } \\
\text { docking }\end{array}$ \\
\hline Position Error H/V [m] & $<10 / \mathrm{NA}$ & $<1 / \mathrm{NA}$ & $<0.1$ \\
\hline Alarm limit [m] & 25 & 2.5 & 0.25 \\
\hline Time to alarm [s] & 10 & 10 & 10 \\
\hline Integrity risk & $1 \mathrm{e}-5 / 3 \mathrm{~h}$ & $1 \mathrm{e}-5 / 3 \mathrm{~h}$ & $1 \mathrm{e}-5 / 3 \mathrm{~h}$ \\
\hline Availability [\%per30days] & 99.8 & 99.8 & 99.8 \\
\hline Continuity [\%over 3h] & NA & 99.97 & 99.97 \\
\hline Coverage & global & local & local \\
\hline
\end{tabular}




\section{ANALYSIS OF STANDARD PNT SENSORS AND INTEGRITY} APPROACHES

\section{A. Sensor analysis}

According to the carriage requirement demanded by IMO Safety of Life at Sea (SOLAS) convention [7], following sensors should be used in maritime applications.

TABLE II. IMO DEMANDED SENSORS FOR PNT UNIT

\begin{tabular}{|c|c|c|}
\hline Sensor & Output & Typical realization \\
\hline \multirow[t]{2}{*}{ Speed log } & STW & $\begin{array}{l}\text { Electromagnetic } \\
\text { logs }\end{array}$ \\
\hline & $\mathrm{SOG}$ & Doppler logs \\
\hline \multirow[t]{2}{*}{ Compass } & True heading & Gyrocompass \\
\hline & $\begin{array}{c}\text { Magnetic } \\
\text { heading }\end{array}$ & Magnet compass \\
\hline EPFS & $\begin{array}{l}\text { Position } \\
\text { SOG } \\
\text { COG } \\
\text { Time } \\
\end{array}$ & $\begin{array}{c}\text { GPS/GLONASS } \\
\text { DGPS/DGLONASS } \\
\text { receiver and } \\
\text { antenna }\end{array}$ \\
\hline $\begin{array}{l}\text { Transmitting Heading Device } \\
\text { (THD) }\end{array}$ & Heading & $\begin{array}{l}\text { GNSS multi- } \\
\text { antenna system }\end{array}$ \\
\hline Echo sounder & UKC & Sonar \\
\hline
\end{tabular}

GNSS has become the most popular sensor for EPFS in the maritime navigation. GNSS, currently GPS and GLONASS offer two positioning possibilities in maritime navigation, the Standard Positioning Service (SPS) and Differential GNSS (DGNSS). Besides the current GPS and GLONASS system, the European Galileo system under development is another promising candidate, which offers two services for maritime community: Safety of Life and Open Service [8].

For maritime application there exist separate standards for GNSS, including: GPS receiver [9], GLONASS receiver[10], GPS/GLONASS combined receiver [11], DGPS and DGLONASS receiver [12], Galileo receiver [8].

These standards are based on their respective GNSS service specifications only. There exists no obvious mapping of these standards to the requirements on the EPFS. Additionally, the option that one GNSS system could serve as redundant system to another GNSS system (e.g. GLONASS $\Leftrightarrow$ GPS) is not discussed in these standards.

Speed logs are the shipborne sensors outputting the speed information. Speed $\operatorname{logs}$ work independently of the radio signals, so that they are used as main sensor for velocity determination. GNSS-based velocity determination can serve as redundant system and enables the integrity monitoring for speed information.

Gyrocompass [13] or magnetic compass [14] is needed to measure the orientation of the ship body in the horizontal plane. Although the true heading obtained from gyrocompasses is normally of great interest in the maritime navigation, magnetic compasses are still popular sensors due to its small size, light weight, flexible installation and independence of power supply. Compasses can be coupled with speed logs to implement the Dead Reckoning (DR) which plays a roll of contingency of GNSS positioning.
THD [15] could obtain heading information from an external system and transfer it to PNT unit. One possible realization of a THD is a GNSS multi-antenna system [16]. Its cost-effectiveness, non-drifted results and acceptable accuracy make it draw more and more attentions in maritime application. However, such a system suffers from the same limitations as GNSS. Besides that, the processing of GNSS carrier phase data is still a challenging task, especially for single-frequency receiver and long-baseline configuration.

Echo sounder [17] uses ultrasonic sound pulses directed down from vessel to measure the distance to the sea bottom, so it is a direct measurement of the UKC and does not rely on an external service.

For the above listed sensors except for GNSS, the selfmonitoring of integrity is still an issue. It will be addressed later that the plausibility tests and validity tests are commonlyused approaches [18]. Nevertheless, the implementation of both tests needs to consider the individual sensor specifications and the properties of the ship, and no standard algorithm is defined.

\section{B. Integrity monitoring}

\section{1) for GNSS}

\section{a) IALA Beacon DGNSS}

GNSS integrity-monitoring services are usually part of augmentation services which also provide DGNSS corrections. The reason for this shared activity is the similarity of the infrastructure required for DGNSS and integrity monitoring. The IALA beacon Differential GNSS service is a standardized technique for maritime use. The competent authority should refer to [19] for recommendations of implementing integrity checks of the DGNSS system.

Currently within the IALA Beacon DGNSS only the integrity of the DGNSS service is monitored, and a flag bit is distributed to indicate whether or not the correction data of this station should be used. Currently, IALA Beacon DGNSS service is not an integrity monitor for the GNSS service itself. For example, it does not check the health status of the satellites in view. However, it could principally be used for that purpose.

For future maritime application, also other augmentation systems could be used for integrity monitoring purpose [5], like e.g. Satellite Based Augmentation Systems (WAAS and EGNOS) or RTK based GBAS systems [20].

\section{b) Receiver Autonomous Integrity Monitoring (RAIM)}

RAIM is a technique whereby the redundant information available at a GNSS receiver is autonomously processed to monitor the integrity of the navigation signals [6]. RAIM has two-fold tasks, first is to check the occurrence of a failure, and second is to identify the satellite(s) in error. RAIM can be done by using the measurement recorded at a single epoch only (so called "snapshot" algorithm) or employing earlier measurement. The first approach needs the redundant measurement, and hence different numbers of satellites in view lead to different performance. In [21], several classic "snapshot" algorithms are introduced. Optimal RAIM 
algorithms should exhibit high detection rates and low false alarm rates [22].

IMO requires that shipborne GNSS receivers should offer an internal integrity test, but it does not specify a standard algorithm and relevant parameters for maritime application.

\section{2) Integrated Naviagtion System (INS)}

An INS is a composed system which provides integrated and augmented functions to support system tasks like collision avoidance, route planning and route monitoring. Currently an INS is not a mandatory system, but if an INS is installed onboard a vessel it is accepted as a replacement of the mandatory single sensors. Integrity monitoring is considered as an intrinsic function of the INS. The currently valid INS standard is based on IMO resolution MSC.86 (70) [23] and is specified within the IEC-61924 standard [24]. A task oriented concept is already introduced in a new resolution MSC. 252 (83) [25], but the specification within the related IEC standard is not yet published. Therefore our analysis is based on IEC61924 standard only. According to IEC-61924 the plausibility check and validity check are required as standard approaches for detecting the gross sensor failure [26]. The plausibility check tests whether the sensor raw data or derived navigational result falls into predefined value range. For example, an output of $181^{\circ}$ from a gyrocompass ranging between $-180^{\circ}$ to $+180^{\circ}$ is not plausible. The validity is tested by comparing the sensor data or derived navigational results with formal and logical criteria, such as whether they fit the ships' maneuver or dynamic properties, or whether they are consistent with the environment nearby. For example, if the output of a speed log is larger than the maximal achievable speed of the ship, a sensor failure can be assured.

Besides plausibility and validity checks, an integrity monitoring based on the following parameters is also required:

(i) Position: comparison with a second EPFS; using RAIM GNSS function; dead reckoning using the ship's heading and speed measuring device

(ii) Heading: comparison with a second heading sensor and a course over ground sensor

(iii) SOG: comparison with a second SOG sensor, with speed through water sensor and with SOG from the EPFS (GNSS)

(iv) Time: comparison with a second time sensor and with the internal INS clock

(v) UKC: comparison with a second depth sensor and with data derived from ships position and electronic navigation charts (ENC)

The threshold for integrity calculations shall be based on the specified or expected (where not specified) sensor accuracy at a probability of $95 \%$ [24].

Comparing the user needs described before with the current standard of the INS the following gaps can be identified:

i. Position, as the most important PNT information, currently needs to be measured by only two separate receiver/antenna GNSS devices. Integrity monitoring is restricted to a comparison of the positions determined by these two receivers. In case of a failure of this
GNSS system no redundant or backup system is available.

ii. Currently onboard a vessel no estimation of positioning error is performed.

iii. As already mentioned, the application of a RAIM is requested, but the algorithms need to be defined. This is a prerequisite for the definition of a further usage of the RAIM output.

iv. The performance requirement on future GNSS is categorized into different operational areas, as shown in Table I. However, in order to really check against these requirements, these areas themselves and the intersection from one area to another need to be clearly marked in the Electronic Navigational Charts.

\section{SELECTION OF SENSORS FOR PNT UNIT}

The selection of sensors used in the integrated PNT unit should take two points into consideration. Firstly, each PNT output should be acquirable from at least one sensor independently (not by sensor integration). Secondly, redundant information for each PNT output is needed to realize the integrity monitoring. Table III shows the PNT output and corresponding sensors. The rows with white background represent the standard sensor, whereas the rows with dark background represent the non-standard sensors which will be introduced in the latter parts.

TABLE III. SENSORS AND OUTPUT

\begin{tabular}{|c|c|c|c|c|c|c|c|}
\hline & Pos & COG & SOG & $\begin{array}{c}\text { True } \\
\text { Heading }\end{array}$ & ROT & Time & UKC \\
\hline $\begin{array}{c}\text { Major GNSS } \\
\text { device }\end{array}$ & $\mathrm{M}$ & $\mathrm{M}$ & $\mathrm{R}$ & & & $\mathrm{M}$ & \\
\hline $\begin{array}{c}\text { Second GNSS } \\
\text { device }\end{array}$ & $\mathrm{R}$ & $\mathrm{R}$ & $\mathrm{R}$ & $\mathrm{R}$ & $\mathrm{B}$ & $\mathrm{R}$ & \\
\hline $\begin{array}{c}\text { Second GNSS } \\
\text { system }\end{array}$ & $\mathrm{R}$ & $\mathrm{R}$ & $\mathrm{R}$ & & & $\mathrm{R}$ & \\
\hline $\begin{array}{c}\text { Second GNSS } \\
\text { signal }\end{array}$ & $\mathrm{R}$ & $\mathrm{R}$ & $\mathrm{R}$ & & & $\mathrm{R}$ & \\
\hline EM Log & & & $\mathrm{B}$ & & & & \\
\hline Doppler Log & & & $\mathrm{M}$ & & & & \\
\hline $\begin{array}{c}\text { Magnet } \\
\text { Compass }\end{array}$ & & & & $\mathrm{B}$ & $\mathrm{B}$ & & \\
\hline Gyrocompass & & & & $\mathrm{M}$ & $\mathrm{B}$ & & \\
\hline THD & & & & & $\mathrm{B}$ & & \\
\hline ROT indicator & & & & & $\mathrm{M}$ & & \\
\hline Echo sounder & & & & & & & $\mathrm{M}$ \\
\hline IMU & $\mathrm{C}$ & $\mathrm{C}$ & $\mathrm{C}$ & $\mathrm{C}$ & $\mathrm{C}$ & & \\
\hline e-Loran & $\mathrm{B}$ & & & & & $\mathrm{B}$ & \\
\hline R-mode & $\mathrm{B}$ & & & & & $\mathrm{B}$ & \\
\hline e-Pelorus & $\mathrm{C}$ & & & $\mathrm{C}$ & & & \\
\hline $\begin{array}{c}\text { Radar(map- } \\
\text { matching) }\end{array}$ & $\mathrm{C}$ & & & & & & \\
\hline TRN & $\mathrm{C}$ & & & & & & \\
\hline
\end{tabular}

R: Redundant B: Backup C: Contingency M: Main sensor

Robust PNT information as required within e-Navigation needs three complementary components of GNSS [3]: (1) a core Global Navigation Satellite System (GNSS); (2) augmentation of GNSS to ensure that GNSS system performance is fit for purpose, including DGNSS. (3) adequate 
backup in the event of GNSS system failure. Using the standard sensors it is difficult to handle GNSS outages, and hence the following techniques can be considered:

\section{A. Carriage of an additional GNSS device}

Once the major GNSS device (antenna or receiver) is out of use, the second GNSS device can fully take the function of the major GNSS device. Using multiple GNSS antennas could enable attitude determination. However, the redundant GNSS device is also affected by the errors related to the radio signal. In this sense, the significance of a redundant GNSS device is reflected during the internal failure of the major GNSS device.

\section{B. Use of two ore more frequency receiver for future GNSS}

GNSS satellites are equipped with more than one signal. Civilian code data will also be encoded into the carrier signal besides L1 signal at future GNSS satellites. The additional civilian code data will offer the same functionalities like the SPS service and therefore can backup the L1 code data. Due to different carrier signals, the other carrier signals might not suffer from the same interference, jamming or spoofing as L1 signal. Also, the channel failure (loss of lock or cycle-slips, etc.) for L1 signal might not occur simultaneously on the other frequencies. When both signals are available the usage of the combination of two frequencies can additionally eliminate the ionospheric error. Nevertheless, errors due to space atmosphere and signal propagation will influence all the carrier signals of a satellite. Hardware failure of receiver or antenna might also challenge the reception of all carrier signals.

\section{Multiple GNSS systems}

Two or more full-operational GNSS could serve as redundancy for each other, as they realize same functions in maritime navigation as specified in [27]. Once one GNSS system is shut down or temporarily out of service, its function can be replaced by another full-operational GNSS system. However, GLONASS and GALILEO are not yet fully operational. Future GNSS receivers and corresponding antennas allow the reception and processing of multiple GNSS signals, however, a hardware failure can cause the loss of all GNSS signals.

\section{Terrestrial navigation system}

Such systems like e-Loran or R-mode facilitate the functions for positioning and e-Loran system can also be used for time determination, so that these systems could serve as backup for GNSS.

\section{1) e-Loran}

e-Loran, the modernized version of Loran-C, is a longrange radio navigation system, operating at an assigned frequency of $100 \mathrm{kHz}$. A position is determined by measuring the time of arrival of signals from at least 3 stations in view [28]. The accuracy of e-Loran is specified in [3] as $8-20 \mathrm{~m}$ where differential e-Loran corrections are provided and smaller than $100 \mathrm{~m}$ elsewhere. Compared to GNSS, e-Loran signals are transmitted at lower frequency with higher power and hence it is not easy to be jammed especially not by the same jammers used for GNSS. It relies on the radio signal propagated over ground and hence does not suffer from the same errors in the propagation path from sky like GNSS. Currently there exists a pre-operational e-Loran network in the UK and Ireland. Whereas the United States have decided to switch off their Loran-C service, operational Loran-C services can be found in parts of Russia and Asia. So the future of e-Loran as a terrestrial backup for GNSS with a large coverage area is currently an open question. Also, the fulfillment of the future maritime requirements with respect to the accuracy is an issue.

\section{2) R-Mode}

In [3], the Ranging-mode (R-mode) is seen as a possible novel variant of positioning technique using terrestrial signals. The idea is to use existing communication channels and append their functionality by sending an additional timing signal. From the time difference between signal transmission and reception, the ship should be able to determine its position. The advantage of this idea would be, that at least partially existing infrastructure could be used. Currently this is still only an idea, where the proof of concept needs to be shown.

\section{E. e-Pelorus}

The positioning relies on the bearing angles obtained from optic sensor with respect to known terrestrial objects. More than two bearing angles allow the horizontal positioning. The use of such a technique needs the objects with known coordinates and simultaneous tracking of several objects. For this purpose, an 'electronic pelorus' is proposed [3]. The independence of radio signal makes it a contingency for GNSS positioning. However, such a technique is still in a design phase and its performance needs to be proved.

\section{F. Radar map-matching}

For this technique the Radar image, containing relevant signature (e.g. coastline), is compared to map stored in the database. By application of a map matching algorithm a determination of a position could be possible. Such a technique does not rely on the radio signal and hence can serve as contingency system for GNSS. However, this technique is relying on a surrounding area which leads to a unique radar signal and hence can only achieve a limited local coverage.

\section{G. Terrain Referenced Navigation (TRN)}

In combination with a precise electronic navigation chart and a speed sensor the determined depth from an echo sounder could also be used for positioning. The independence of radio signal makes it a contingency system for GNSS. However, it relies on precision of the chart and the topographic conditions of the sea bottom. 


\section{H. Inertial Measurement Unit (IMU)}

The development in the field of inertial sensors, with increasing quality of rather low cost sensors, makes inertial navigation attractive for future civil maritime applications. Introducing an IMU into the PNT unit offers at least the following advantages: (1) It neither receives nor emits detectable radiation and requires no external antenna, and hence it is immune to signal jamming and inherently stealthy. (2) IMU can bridge GNSS outage within certain duration. From this point of view, IMU is a short-term contingency for the navigation. (3) In case that GNSS signals suffer from large multipath error or low signal to noise ratio, IMU can still measure accurate short-term relative movement to improve the positioning accuracy. (4) IMU could offer the functions of the mandatory sensors like logs, gyrocompass, rate-of-turn indicator. (5) IMU can provide high output rate if GNSS receiver is working under low data rate. (6) IMU allows the integrity monitoring of on-board sensor for these outputs. (7) The price of IMU at industrial and tactical level is quickly falling down. The significant limitations of IMU in maritime navigation lie in the following aspects. (1) The position, velocity and attitude accuracy obtained from IMU will be degraded with time due to the sensor bias and numerical integrations, so that long-term stability is still an issue. (2) The continuous vibration of the ships challenges the initialization of the IMU.

In an IMU, the biases of gyroscopes and accelerometers, as well as the drift due to the integration need to be identified by integrating IMU with other sensors. The integration of IMU and GNSS using Kalman filter is the most popular integration scheme. It is also the basis for expending the integration to other shipborne sensors. For maritime navigation, looselycoupled and tightly-coupled integration strategies can be applied [29]. An advantage comparison between both architectures is given in Table IV.

TABLE IV. ADVANTAGES OF DIFFERENT INTEGRATION ARCHITECTURES

\begin{tabular}{|c|c|}
\hline $\begin{array}{c}\text { Advantages of loosely-coupled } \\
\text { integration }\end{array}$ & $\begin{array}{c}\text { Advantages of tightly-coupled } \\
\text { integration }\end{array}$ \\
\hline $\begin{array}{l}\text { (1) faster responds to the IMU errors } \\
\text { [30] } \\
\text { (2) easy implementation for } \\
\text { switching positioning models (for } \\
\text { example, switch from DGNSS to } \\
\text { SPS and vice versa) }\end{array}$ & $\begin{array}{l}\text { (1) integrity monitoring for each } \\
\text { GNSS measurement } \\
\text { (2) working under less than } 4 \\
\text { satellites in view }\end{array}$ \\
\hline
\end{tabular}

\section{INTEGRITY FOR PNT UNIT}

For a PNT unit, integrity monitoring can be carried out in three sequential steps. The first step is individual sensor data test. The second step is the compatibility test of similar data from different sensors. The third step is the fault detection and identification in the integration algorithm. A general integrity monitoring approach is depicted in Figure I. The first step is already elaborated in the previous parts. The second and third steps will be briefly introduced in the following parts:

\section{A. Compatibility test of sensor data}

Once a specific output can be provided by more than one sensor, different sensor data can be compared to perform the compatibility test. A significant discrepancy between different sensors implies the failure of at least one of these sensors. The upper bound for deviation should be defined either a priori or in real-time according to the previous measurements. From Table III it can be seen that most outputs can be provided by more than one sensor, so that the compatibility test is possible. The compatibility test should be carried out before sending the sensor data to integration algorithm.

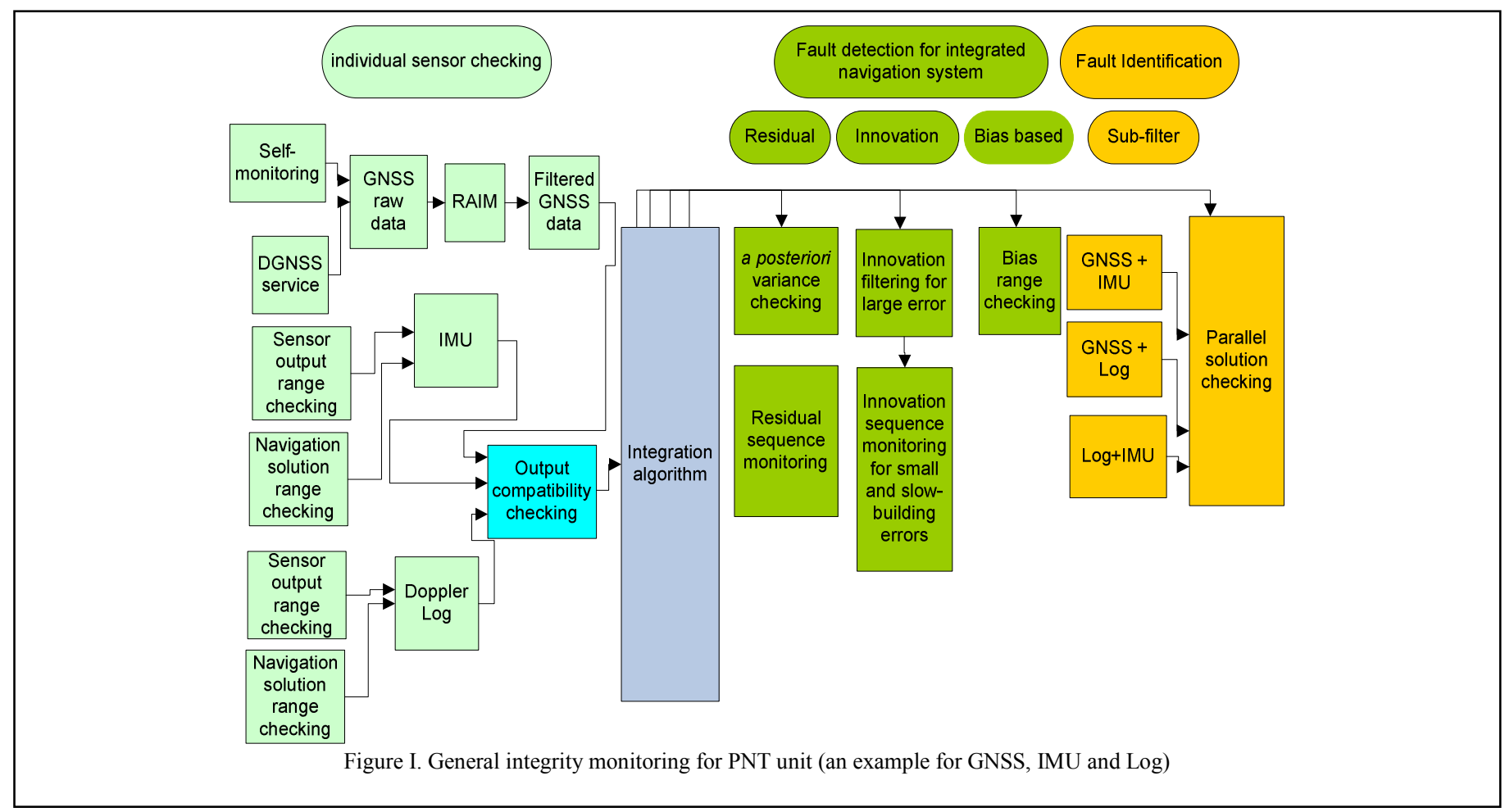




\section{B. Fault detection for integrated navigation system}

The plausibility tests, validity tests and compatibility tests are suitable for detecting gross sensor failure but not sensitive for slight error, time-variant errors and drifts. The Kalman filter-based algorithm could offer high sensitivity of detecting these errors. Integrity monitoring based on Kalman filter can be categorized into the following approaches [31].

\section{1) Kalman filter estimates (bias check)}

In a Kalman filter, the sensor measurement biases can be estimated. If an estimated bias is significantly larger than the error level specified by the manufacturer, there is likely to be a failure in the sensor.

\section{2) Innovation-based approaches}

The innovations indicate the consistency of the actual measurements and the measurements predicated by state estimates. Innovation filtering may be used to detect large discrepancies immediately, whereas innovation sequence monitoring enables smaller discrepancies to be detected over time.

\section{3) Residual-based approaches}

The above-mentioned innovation filtering and sequence monitoring can also be expanded to residuals. Residuals have a smaller covariance than innovation, making them more sensitive for error detection [31]. The only shortcoming is that the processing of residuals is not an essential part of a Kalman filter routine and needs extra computing time.

Another popular approach related to residuals is the DIA (Detection, Identification and Adaption) approach [32], which is based on the a posteriori variance factor for a least-squares adjustment.

\section{4) Parallel solution of multiple sub-filters}

Parallel-solutions integrity monitoring maintains a number of parallel navigation solutions or sub-filters, each excluding data from one sensor or radio navigation signal. Each additional navigation solution is compared with the main filter using a consistency test. A significant inconsistency indicates a fault in the sensor or signal omitted from main filter. The system output is then switched to the solution omitting the faulty sensor or signal. The main drawback lies in the increased computational burden and hence this technique is preferably used for failure identification rather than failure detection.

\section{SUMMARY \& CONCLUSIONS}

This paper focuses on a maritime integrated PNT unit as a possible replacement of the current mandatory EPFS onboard a vessel. We interpret a PNT unit as a system function, which collects and integrates data from individual PNT sensors in order to provide PNT information with a specified accuracy and integrity. The PNT unit should be based on a modular concept, though it could contain different sensors for different vessel types as it is required by IMO. For vessels equipped with an INS, it can be seen as a part of the INS to deliver robust PNT information. It should internally identify and automatically switch to the sensors or sensor combinations delivering the best PNT solution.
Focusing on integrity for PNT information we have analyzed the current state of the art sensors and related maritime standards. Here we found a need of defining accuracy and integrity requirements for all parameters (e.g. position, SOG, COG) deduced from user requirements according to the different operational areas and/or volume of traffic and level of risk.

Subsequently we have discussed additional sensors providing redundancy, backup or contingency functionality to the primary GNSS sensor. In this discussion one should not only consider terrestrial radio navigation systems but also the possible future usage of a second GNSS frequency and a second GNSS system. The development in the field of inertial sensors, with increasing quality of rather low cost sensors, makes inertial navigation attractive for future civil maritime applications. From our prospective inertial navigation could not only provide contingency functionality but could be a key enabler for an efficient integrity monitoring.

As starting point towards a concept for the integrated PNT unit we have analyzed most relevant integrity monitoring approaches.

Finally one has to state, that this paper can only be seen as a first step in the development of an integrated maritime PNT unit which can be one component in order to provide the mariners in the future with more reliable PNT information.

\section{REFERENCES}

[1] IMO, NAV 53/13 Development of an e-Navigation Strategy. 2007.

[2] IMO, NAV 54/25 Annex 12 Draft strategy for the development and implementation of e-Navigation. 2008.

[3] IALA, World Wide Radio Navigation Plan, Edition 1. 2009.

[4] IMO, NAV 55/WP.5 Development of an e-Navigation Strategy Implementation Plan. 2009.

[5] IALA, Recommendation R-129: On GNSS Vulnerability and Mitigation Measures Edition 2. 2008.

[6] IMO, Resolution A.915(22): Revised Maritime Policy and Requirements for A Future Global Navigation Satellite System (GNSS). 2001.

[7] IMO, SOLAS Chapter V: Safety of Navigation. 2002.

[8] IMO, Resolution MSC.233(82): Adoption of the Performance Standards for Shipborne Galileo Receiver Equipment. 2006.

[9] IMO, Resolution MSC.112(73): Adoption of the Revised Performance Standards for Shipborne Global Positioning System (GPS) Receiver Equipment. S. December 2000.

[10] IMO, Resolution MSC.113(73): Adoption of the Revised Performance Standards for Shipborne GLONASS Receiver Equipment. 2000.

[11] IMO, Resolution MSC.115(73): Adoption of the Revised Performance Standards for Shipborne GPS/GLONASS Combined Receiver Equipment. 2000.

[12] IMO, Resolution MSC.114(73): Adoption of the Revised Performance Standards for Shipborne DGPS and DGLONASS Maritime Radio Beacon Receiver Equipment. 2000.

[13] IMO, Resolution A.424(XI): Performance Standards for Gyro Compasses. 1979.

[14] IMO, Resolution A.382(X): Magnetic Compasses Carriage and Performance Standards. 1977.

[15] IMO, Resolution MSC.116(73): Performance Standards for Marine Transmitting Heading Devices (THDs). 2000.

[16] G. Lu, "Performance analysis of a shipborne gyrocompass with a multi-antenna GPS system," presented at the Position Location and Navigation Symposium, 1994., IEEE, Las Vegas, NV , USA, 1994

[17] IMO, Resolution A.224(VII): Performance Standards for EchoSounding Equipment. 
[18] IEC, Maritime Navigation and Radiocommunication Equipment and Systems - Global Navigation Satellite Systems (GNSS) - Part 1:

Global Positioning System (GPS) Receiver Equipment, Performance Standards, Methods of Testing and Required Test Results.

[19] IALA, Recommendation R-121: The Performance and Monitoring Of DGNSS Services in the Frequency Band 283.5 - 325 KHz. 2004.

[20] D. Minkwitz und S. Schlueter, "Integrity Assessment of a Maritime Carrier Phase Based GNSS Augmentation System," presented at the ION GNSS 2010, Portland, USA, 2010.

[21] B. W. Parkinson und J. J. Spiker, Global Positioning System: Theory and Applications: Volume II. American Institute of Aeronautics \& Astronautics, 1996.

[22] S. Hewitson und J. Wang, "GNSS receiver autonomous integrity monitoring (RAIM) performance analysis," GPS Solutions, Bd. 10 , Nr. 3, S. 155-170, Juli. 2006.

[23] IMO, MSC.86(70): Adoption of new and amended performance standards for navigational equipment. 1998.

[24] IEC, IEC 61924 Maritime navigation and radiocommunication equipment and systems - Integrated navigation systems - Operational and performance requirements, methods of testing and required test results. 2006.

[25] IMO, Resolution MSC.252(83): Adoption of the Revised Performance Standards for Integrated Navigation Systems (INS). 2007.

[26] IEC, Maritime navigation and radiocommunication equipment and systems - Integrated navigation systems, Operational and performance requirements, methods of testing and required test results. 2006.

[27] IMO, Resolution A.953(23): World-Wide Radio Navigation System.

[28] IMO, Resolution A.818 (19): Recommendation on Performance Standards for Shipborne Loran-C and Chayka Receivers.

[29] E. D. Kaplan, Understanding GPS Principles and Applications. Artech House Publishers, 1996.

[30] J. D. Gautier, "GPS/INS generalized evaluation tool (GIGET) for the design and testing of integrated navigation systems," Stanford University, 2003.

[31] P. D. Groves, Principles of GNSS, Inertial, and Multi-Sensor Integrated Navigation Systems (GNSS Technology and Applications). Artech House Publishers, 2007.

[32] P. Teunissen und A. Kleusberg, GPS for Geodesy. Springer, 1998. 